\title{
Assessment of Heart Rate Variability in Hypertensive Patients
}

\author{
Archana 1 , Preeti Agarwal ${ }^{2}$ \\ ${ }^{1}$ Associate Professor, Department of Physiology, Subharti Medical College, Meerut, ${ }^{2}$ Professor \& HOD, Department of Anaesthesia, Mulayam Singh Medical \\ College, Merrut
}

\section{Abstract}

Background: Heart rate variability (HRV) has emerged as a practical, noninvasive tool to quantitatively investigate cardiac autonomic dysregulation in hypertension. The present study was conducted to assess heart rate variability in hypertensive patients. Subjects and Methods: The present study was conducted on 45 hypertensive patients of both genders. Spectral indices of HRV such as total power (TP), normalized low frequency power (LFnu), normalized high frequency power (HFnu), ratio of low frequency power to high frequency power (LF-HF ratio), standard deviation of normal-to-normal RR intervals (SDNN), root mean square successive difference (rMSSD) and the proportion of NN50 to the total number of NN intervals (pNN50) were assessed. Results: Group I had 25 males and 20 females while group II had 23 males and 22 females. SDNN (ms) in group I was 157.2 and in group II was 146.3, rMSSD (ms) in group I was 44.5 and in group II was 38.5, pNN50 in group I was 15.4 and in group II was 10.8. LFnu in group I was 76.2 and in group II was 89.1, HFnu in group I was 57.4 and in group II was 37.6, LF/HF ratio in group I was 2.87 and in group II was 4.85. Conclusion: HRV can be used as a routine screening test to predict the future risk of hypertension at an earlier stage. There is an increased sympathetic activity and a decreased vagal tone associated with hypertension.

Keywords: Heart rate variability, sympathetic, Hypertension.

Corresponding Author: Dr. Archana, Associate Professor, Department of Physiology, Subharti Medical College, Meerut.

Received: September 2019

Accepted: September 2019

\section{Introduction}

Blood pressure is maintained physiologically by multiple regulatory mechanisms such as neural control, hormonal control and local control mechanism. Among them, neural control by Autonomic nervous system (ANS) is the most important regulatory mechanism of blood pressure. Though hypertension is a multifactorial disease, ANS dysfunction is an important factor in the development and progression of hypertension. ${ }^{[1]}$

Hypertension is defined as a persistent elevated blood pressure of $\geq 140 / 90 \mathrm{mmHg}$. It is one of the most prevalent diseases worldwide. The prevalence of hypertension in South Indian population is around $22.1 \%{ }^{[2]}$ There are many modifiable risk factors underlying hypertension such as increased body weight, reduced physical activity, high salt intake, alcohol consumption and low potassium intake. Hypertension is a major risk factor for many cardiovascular diseases like coronary artery disease, stroke, heart failure and end-stage renal disease. It has been estimated that hypertension accounts for $6 \%$ of death worldwide. Thus, primary prevention of hypertension may reduce the overall risk of cardiovascular diseases. ${ }^{[3]}$

Heart rate variability (HRV) has emerged as a practical, noninvasive tool to quantitatively investigate cardiac autonomic dysregulation in hypertension. ${ }^{[4]}$ Studies have reported decreased HRV among hypertensives and that the relation between blood pressure and HRV is present across a wide range of blood pressures. ${ }^{[5]}$ Data from the Framingham cohort and a subset of the Atherosclerosis Risk in Communities (ARIC) cohort suggest that individuals with decreased HRV have an increased risk of developing hypertension, although results are inconsistent across measures of HRV and sex. It is also unknown to what degree hypertensives and normotensive experience similar declines in HRV. Thus, although the autonomic nervous system is involved in the regulation of blood pressure, the temporal sequence linking hypertension and HRV is unclear. ${ }^{[6]}$ The present study was conducted to assess heart rate variability in hypertensive patients.

\section{Subjects and Methods}

The present study was conducted in the department of Physiology. It comprised of 45 hypertensive patients of both genders. Equal number of normotensive subjects was also included. All were informed regarding the study and written consent was obtained. Ethical clearance was obtained from institutional ethical committee.

General information such as name, age, gender etc, was recorded. A thorough clinical examination was done. In all subjects, blood pressure measurement was done following auscultatory method. Group I comprised of hypertensive patients and group II had normotensive subjects. The criteria for diagnosing hypertension were BP $\geq 140 / 90$ $\mathrm{mmHg}$ based on the average of 3 consecutive readings at an interval of 3 weeks. Subjects with blood pressure values of 
100-119/60-79 mm Hg were recruited as normotensive. Spectral indices of HRV such as total power (TP), normalized low frequency power (LFnu), normalized high frequency power (HFnu), ratio of low frequency power to high frequency power (LF-HF ratio), standard deviation of normal-to-normal RR intervals (SDNN), root mean square successive difference (rMSSD) and the proportion of NN50 to the total number of NN intervals (pNN50) were assessed. Results were tabulated and subjected to statistical analysis. $P$ value less than 0.05 was considered significant.

\section{Results}

Table 1: Distribution of subjects

\begin{tabular}{|l|l|l|}
\hline Gender & Group I (Hypertensive) & Group II (Normotensive) \\
\hline Males & 25 & 23 \\
\hline Females & 20 & 22 \\
\hline
\end{tabular}

[Table $1 \&$ Figure 1] shows that group I had 25 males and 20 females while group II had 23 males and 22 females.

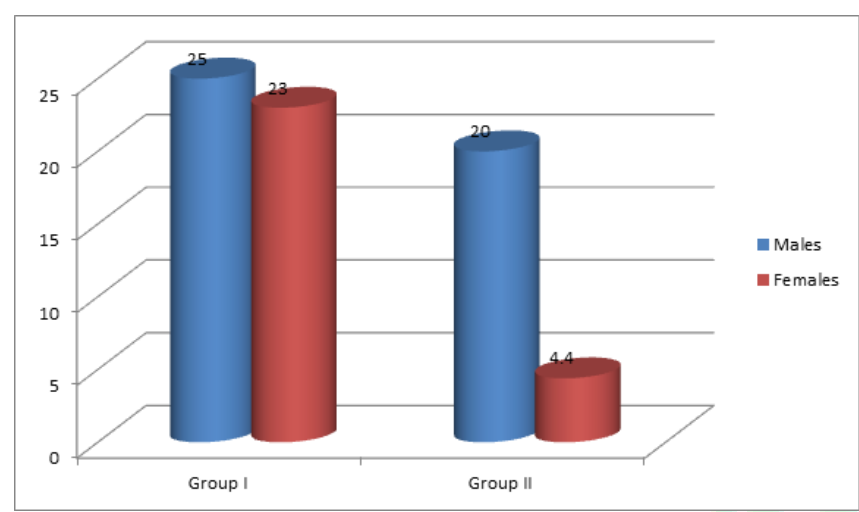

Figure 1: Distribution of subjects

Table 2: Assessment of time domain

\begin{tabular}{|l|l|l|l|}
\hline Time domain & Group I & Group II & P value \\
\hline SDNN (ms) & 157.2 & 146.3 & 0.02 \\
\hline rMSSD $(\mathrm{ms})$ & 44.5 & 38.5 & 0.05 \\
\hline pNN50 & 15.4 & 10.8 & 0.02 \\
\hline
\end{tabular}

[Table $2 \&$ Figure 2] shows that SDNN (ms) in group I was 157.2 and in group II was 146.3, rMSSD (ms) in group I was 44.5 and in group II was 38.5, pNN50 in group I was 15.4 and in group II was 10.8 .

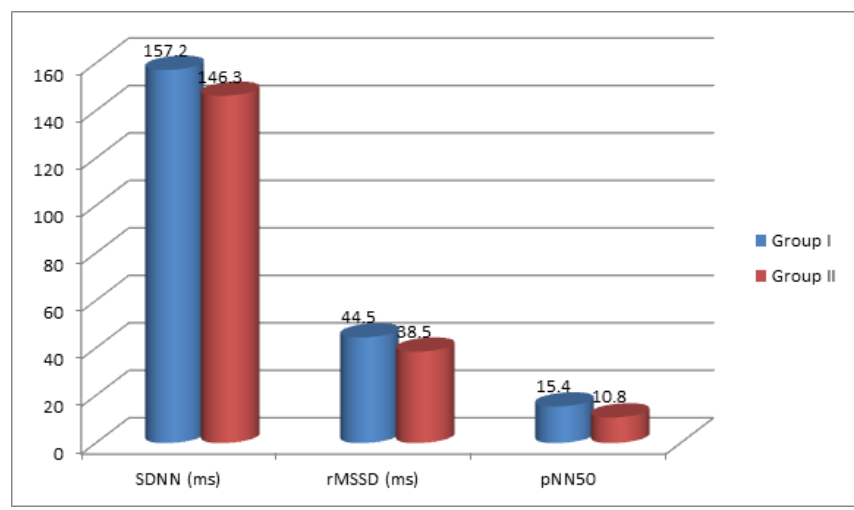

Figure 2: Assessment of time domain
Table 3: Assessment of frequency domain

\begin{tabular}{|l|l|l|l|}
\hline Time domain & Group I & Group II & P value \\
\hline LFnu & 76.2 & 89.1 & 0.05 \\
\hline HFnu & 57.4 & 37.6 & 0.01 \\
\hline LF/HF ratio & 2.87 & 4.85 & 0.01 \\
\hline
\end{tabular}

[Table 3 \& Figure 3] shows that LFnu in group I was 76.2 and in group II was 89.1, HFnu in group I was 57.4 and in group II was 37.6, LF/HF ratio in group I was 2.87 and in group II was 4.85 .

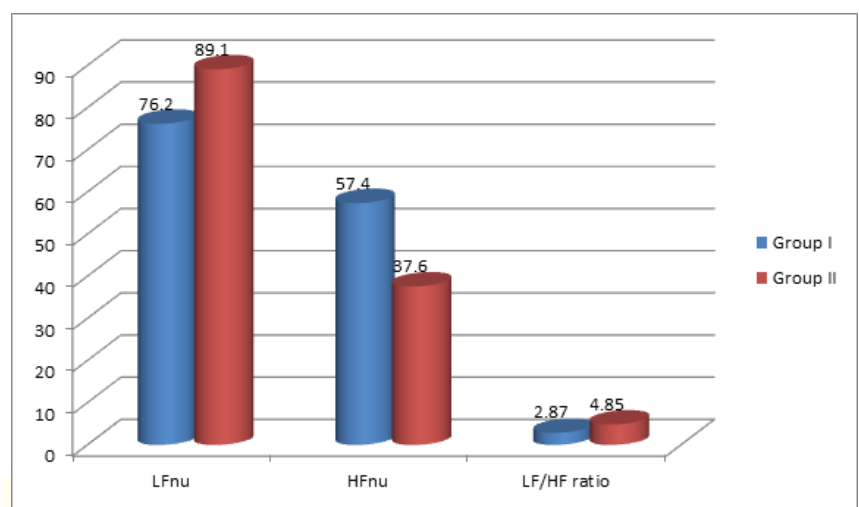

Figure 3: Assessment of frequency domain

\section{Discussion}

The autonomic nervous system (ANS) plays a fundamental role in the control of arterial blood pressure and heart rate, and, therefore, may be considered an important pathophysiologic factor in the development of arterial hypertension. ${ }^{[7]}$ Currently, the status of autonomic action of the heart may be known through the study of heart rate variability. ${ }^{[8]}$ Heart rate varies per beat as a consequence of the constant adaptations promoted by the ANS to maintain cardiovascular system balance. These alterations may be assessed through the variations in R-R intervals, therefore, constituting the heart rate variability. ${ }^{[9]}$ The integration between the sympathetic and parasympathetic modulations determines heart rate variability. As a research tool, assessment of heart rate variability has provided a better understanding of the participation of the ANS in different physiological and pathological situations of the cardiovascular system. The assessment of heart rate variability has stimulated a large number of observations, indicating the potential value of that approach in the diffusion of knowledge about the alterations in the mechanisms of blood pressure control involved in hypertension. ${ }^{[10]}$ The present study was conducted to assess heart rate variability in hypertensive patients.

In present study, group I had 25 males and 20 females while group II had 23 males and 22 females. Xie et al, ${ }^{[11]}$ conducted a study to assess the sympathovagal balance by analysing the HRV changes in hypertensive individuals. The study was conducted on 30 normotensive and 30 hypertensive subjects $(\mathrm{BP} \geq 140 / 90 \mathrm{~mm} \mathrm{Hg}$, according to JNC-7 classification). Results showed significantly reduced HFnu $(p<0.05)$, SDNN $(p<0.05), \operatorname{rMSSD}(\mathrm{p}<0.05)$ and 
pNN50 $(\mathrm{p}<0.05)$ in hypertensive individuals. LFnu and LFHF ratio was significantly increased $(p<0.05)$ in hypertensive individuals.

We found that SDNN (ms) in group I was 157.2 and in group II was 146.3 , rMSSD (ms) in group I was 44.5 and in group II was 38.5, pNN50 in group I was 15.4 and in group II was 10.8. We found that SD LFnu in group I was 76.2 and in group II was 89.1, HFnu in group I was 57.4 and in group II was 37.6, LF/HF ratio in group I was 2.87 and in group II was 4.85 .

Menezes et al, ${ }^{[12]}$ conducted a study comprised 286 patients diagnosed with arterial hypertension ( $\mathrm{AH}$ ) for the first time and divided into 4 groups according to diastolic blood pressure (DBP) levels: group A - DBP $<90 \mathrm{mmHg}$; group B - DBP 90-99 mmHg; group C - DBP 100-109 mmHg; group D - DBP>110 mmHg. Group A (110 healthy individuals) and group $\mathrm{C}$ (79 patients with moderate $\mathrm{AH}$ ) underwent 24-hour Holter-ECG with analysis of heart rate variability in time domain (TD) and frequency domain (FD). The group $\mathrm{C}$ patients were treated with ACE inhibitors for 3 months, and, after this period, they underwent a new 24-hour Holter- ECG study for assessing heart rate variability, the values being compared with those of normotensive individuals. The SDNN and PNN50 parameters (TD), and the LF spectrum (FD) were significantly different in the 2 groups, with clearly reduced values in hypertensive individuals $(\mathrm{P}<0.05)$. Group $\mathrm{C}$ patients, after treatment with ACE inhibitors, showed a recovery in all variables of heart rate variability, achieving values close to those of normotensive individuals.

\section{Conclusion}

HRV can be used as a routine screening test to predict the future risk of hypertension at an earlier stage. There is an increased sympathetic activity and a decreased vagal tone associated with hypertension.

\section{References}

1. Michel Slama, Dinko Susic, Edward D Frohlich. Prevention of hypertension. Current Opinion in Cardiology 2002; 17: 531-536

2. Kotchen TA. Hypertensive vascular disease. Harrison's principle of internal medicine 2008. 17th edition Mc Graw Hills; Chapter 241: $1549-62$

3. Tolga Dogru M, Vedat Simsek, Omer sahin. Differences in autonomic activity in individuals with optimal, normal and high-normal blood pressure levels. Arch Turk Soc Cardiol 2010; 38: 182-188

4. Havlicekova Z, Tonhajzerova I, Jurko Jr A, Jesenak M, Durdik P, Nosal S, Zelenak K, Antosova M , Banovcin P. Cardiac autonomic control in adolescents with primary hypertension. Eur J Med Res 2009; 14: 101-103

5. Nasim Kari, Jahan Ara Hasan and Syed Sanowar Ali. Heart rate variability - a review. Journal of Basic and Applied Sciences 2011; 7: 71-77

6. Farah khaliq, Keshav gupta, Pawan singh. Autonomic reactivity to cold pressor test in prehypertensive and hypertensive medical students. Indian J Physiol Pharmacol 2011; 55: 246-252

7. Chobanian AV, Bakrish GL, Black HR, Cushman WC et al. Seventh report of the joint National Committee on prevention, detection, evaluation \& treatment of High Blood Pressure, J Hypertension 2003; 42: 1206-125.

8. Pal GK, Pravati Pal. Spectral analysis of Heart Rate Variability. Textbook of Practical Physiology 2010.3rd edition Universities Press ; Chapter :

9. Jagmeet P, Martin G Larson, Hisako Tsuji, Jane C Evans, Christopher JO Donnell, Daniel Levy. Reduced Heart rate variability and newonset Hypertension. Hypertension 1998; 32: 293-297.

10. Virtanen R, Jula RA, Kuusela T, Helenius H, Voipio Pulkki LM. Reduced Heart rate variability in Hypertension. Journal of Human Hypertension 2003; 17: 171-179.

11. Xie Gui-Ling, Wang Jing-hua, Zhou Yan, Xu Hui, Sun Jing-Hui, Yang Si-Rui. Association of High Blood Pressure with Heart Rate Variability in Children. Iran J Paediatr 2013; 23: 37-44.

12. Mohd Urooj, Pillai KK, Monika Tandon, Venkateshan SP, Nilanjan Saha. Reference ranges for time domain parameters of Heart rate variability in Indian population and validation in hypertensive subjects and smokers. Int J Pharm Pharm Sci; 2011;3(1): 36-39.

13. Menezes JR, Oliveira LLM, Melo CSN, Freitas JR. Heart rate variability and Autonomic nervous system response in Hypertensive patients with and without ACE inhibitors. Progress in Biomedical research 2000; $1: 385-388$

Copyright: (C) the author(s), 2019. It is an open-access article distributed under the terms of the Creative Commons Attribution License (CC BY 4.0), which permits authors to retain ownership of the copyright for their content, and allow anyone to download, reuse, reprint, modify, distribute and/or copy the content as long as the original authors and source are cited.

How to cite this article: Archana, Agarwal P. Assessment of Heart Rate Variability in Hypertensive Patients. Asian J. Med. Res. 2019;8(3):PH01-PH03.

DOI: dx.doi.org/10.21276/ajmr.2019.8.3.PH1

Source of Support: Nil, Conflict of Interest: None declared. 